\title{
O ENFERMEIRO NO PROGRAMA DE GERENCIAMENTO DO USO DE ANTIMICROBIANOS: UMA REVISÃO INTEGRATIVA
}

\author{
The nurse in the antimicrobial stewardship: an integrative review
}

El enfermero en el programa de gestión del uso de antimicrobianos: una revisión integratival

André Luiz Silva Alvim ${ }^{1 *}$ (D)

RESUMO: Objetivo: Analisar como a literatura científica descreve o enfermeiro, bem como seu papel no Programa de Gerenciamento do Uso de Antimicrobianos (PGUA). Método: Revisão integrativa da literatura, realizada mediante consulta às bases de dados Scientific Electronic Library Online (SciELO), Literatura Latinoamericana e do Caribe em Ciências da Saúde (LILACS) e Medical Literature Analysis and Retrieval System Online (MEDLINE). Resultados: Foram incluídos seis artigos que atenderam os critérios de inclusão previamente estabelecidos. Todos foram publicados na língua inglesa e divulgados na base de dados eletrônica MEDLINE. A metade dos artigos foi publicada em revistas científicas que tiveram fator de impacto avaliado acima de 2,0, destacando os periódicos Clinical Infectious Diseases (8,216), BMC Medical Informatics and Decision Making (2,288) e American Journal of Infection Control (2,209). Quanto aos temas abordados, todos os artigos reconheceram o enfermeiro como peça fundamental no PGUA. Conclusão: Embora os estudos na literatura sejam limitados, foi possível concluir que a participação do enfermeiro promove otimização terapêutica e uso racional dos antibióticos, contribuindo para redução da resistência antimicrobiana.

Palavras-chave: Equipe de enfermagem. Gestão de antimicrobianos. Infecção hospitalar.

ABSTRACT: Objective: To analyze how scientific literature describes the nurse, as well as his/her role in the Antimicrobial Stewardship (AMS). Method: Integrative literature review, carried out by consulting the Scientific Electronic Library Online (SciELO), Latin American \& Caribbean Health Sciences Literature (LILACS), and MEDical Literature Analysis and Retrieval System Online (MEDLINE) databases. Results: We included six articles that met the previously established inclusion criteria. They were published in English and disclosed in the MEDLINE electronic database. Half of the articles were published in scientific journals that had an impact factor evaluated above 2.0. The journals Clinical Infectious Diseases (8.216), BMC Medical Informatics and Decision Making (2.288), and American Journal of Infection Control (2.209) were highlights. Regarding the addressed themes, all the articles recognized the nurse as a key element in the AMS. Conclusion: Even though the studies in the literature are limited, we concluded that the participation of nurses promotes therapeutic optimization and rational use of antibiotics, contributing to the reduction of antimicrobial resistance. Keywords: Nursing, team. Antimicrobial stewardship. Cross infection.

RESUMEN: Objetivo: Analizar cómo la literatura científica describe el enfermero, así como su papel en el Programa de Gestión del Uso de Antimicrobianos (PGUA). Método: Revisión integrativa de la literatura, realizada mediante consulta a las bases de datos Scientific Electronic Library Online (SciELO), Literatura Latinoamericana y del Caribe en Ciencias de la Salud (LILACS) y Medical Literature Analysis and Retrieval System Online (MEDLINE). Resultados: Fueron incluidos seis artículos que atendieron a los criterios de inclusión previamente establecidos. Todos fueron publicados en el idioma inglés y divulgados en la base de datos electrónica MEDLINE. Mitad de los artículos fue publicada en revistas científicas que tuvieron factor de impacto evaluado arriba de 2,0, destacando los periódicos Clinical Infectious Diseases (8,216), BMC Medical Informatics and Decision Making (2,288) y American Journal of Infection Control $(2,209)$. Con relación a los temas abordados, todos los artículos reconocieron al enfermero como pieza fundamental en el PGUA. Conclusión: Aunque los estudios en la literatura sean limitados, fue posible concluir que la participación del enfermero promueve optimización terapéutica y uso racional de los antibióticos, contribuyendo para la reducción de la resistencia antimicrobiana. Palabras clave: Grupo de enfermería. Programas de optimización del uso de los antimicrobianos. Infección hospitalaria.

'Enfermeiro; mestre em Enfermagem pela Universidade Federal de Minas Gerais (UFMG); especialista em Controle de Infecção Hospitalar; MBA em Auditoria e Gestão da Qualidade aplicada à Serviços de Saúde. Doutorando em Enfermagem pela UFMG - Belo Horizonte (MG), Brasil.

*Autor correspondente: andrealvim1 @hotmail.com

Recebido: 14/11/2018 - Aprovado: 12/06/2019

DOI: 10.5327/Z1414-4425201900030007 


\section{INTRODUÇÃO}

A Resistência Antimicrobiana (RA) diz respeito à capacidade do microrganismo de suportar os efeitos da ação de antibióticos previamente indicados. Esse fenômeno é considerado um problema de saúde pública, que compromete a efetividade dos tratamentos anti-infecciosos atuais. Destacam-se vários fatores que contribuem para a emergência do fenômeno, em especial, o uso de antimicrobianos sem indicação adequada ${ }^{1}$.

Nos Estados Unidos, estima-se que 2 milhões de Infecções Relacionadas à Assistência à Saúde (IRAS) ocorram anualmente por microrganismos resistentes a antimicrobianos e pelo menos 23 mil óbitos ocorrem como resultado dessas infecções ${ }^{1}$. Na Europa, cerca de 25 mil pacientes morrem por infecções causadas por microrganismos multirresistentes, gerando custos adicionais para os cofres públicos de aproximadamente 1,5 bilhão de euros por ano ${ }^{2}$. Já no Brasil, a resistência aos antibióticos vem sendo reportada por diversos serviços de saúde nas últimas décadas ${ }^{3}$.

No contexto nacional, a Portaria $\mathrm{n}^{\circ} 2.616$, de 12 de maio de 1998, estabeleceu diretrizes e normas para criação dos Programas de Controle de Infecções Hospitalares (PCIH) e incluiu uma política obrigatória de utilização de antimicrobianos, germicidas e materiais médico-hospitalares ${ }^{4}$. Ao mesmo tempo, não apenas no Brasil, mas mundialmente, por meio da Organização Mundial da Saúde (OMS) ${ }^{5}$ e do Centro de Prevenção e Controle de Doenças dos Estados Unidos da América, a temática sobre o gerenciamento do uso de antimicrobianos ganhou ampla discussão e destaque entre os temas relacionados à saúde pública ${ }^{6}$.

O Programa de Gerenciamento do Uso de Antimicrobianos (PGUA), do inglês Antimicrobial Stewardship, estabelece diretrizes e estratégias pautadas em evidências científicas e dados laboratoriais para criação de ações sistemáticas que possam prevenir e controlar a disseminação de microrganismos multirresistentes. O PGUA, implantado de forma eficaz, favorece melhores desfechos clínicos, reduzindo o tempo de permanência hospitalar, a morbimortalidade e os custos assistenciais não esperados decorrentes dos tratamentos anti-infecciosos inadequados ${ }^{6-8}$.

Entre os diversos profissionais que compõem a equipe responsável pelo PGUA, destacam-se os enfermeiros. Estes são responsáveis por assegurar que as culturas bacteriológicas sejam realizadas antes do início dos antibióticos, por promover discussões sobre possíveis efeitos adversos causados pelos antimicrobianos e por revisar as prescrições médicas diariamente, conforme o tratamento indicado e a duração do mesmo ${ }^{8}$.
Nesse contexto, este estudo justifica-se por elucidar o papel do enfermeiro frente ao PGUA, visto que algumas literaturas ainda não o reconhecem como peça-chave para o sucesso do programa. Esta pesquisa é importante para fornecer dados que chamem a atenção dos gestores em saúde sobre a importância da enfermagem no combate à resistência antimicrobiana $\mathrm{e}$ espera-se que haja novas reflexões acerca da temática proposta.

\section{OBJETIVO}

Analisar como a literatura científica descreve o enfermeiro, bem como seu papel no Programa de Gerenciamento do Uso de Antimicrobianos.

\section{MÉTODO}

Trata-se de um estudo bibliográfico, do tipo revisão integrativa da literatura. Esse método permite unificar os resultados com base em uma temática definida e incorpora as evidências na prática clínica, contribuindo para o aprofundamento do conhecimento do tema investigado. ${ }^{9}$

Para a elaboração do estudo, as seguintes etapas foram percorridas:

- estabelecimento da hipótese ou questão da pesquisa;

- amostragem ou busca na literatura;

- categorização dos estudos;

- avaliação dos estudos incluídos na revisão;

- interpretação dos resultados;

- síntese do conhecimento ou apresentação da revisão?.

Na primeira etapa, foi definido o tema e os objetivos de pesquisa. Como questão norteadora, formulou-se a seguinte pergunta: Qual é o papel do enfermeiro frente ao Programa de Gerenciamento do Uso de Antimicrobianos?

Para a seleção dos estudos, foi realizado levantamento bibliográfico nas bases de dados Scientific Electronic Library Online (SciELO), Literatura Latino-americana e do Caribe em Ciências da Saúde (LILACS) e Medical Literature Analysis and Retrieval System Online (MEDLINE), por intermédio da Biblioteca Virtual de Saúde (http:/ /bvsalud.org/). Para estratégia de recuperação da informação científica, utilizou-se os operadores booleanos. As palavras-chave definidas para a busca literária foram: "Nurse" AND "Antimicrobial Stewardship" AND hospital, sendo a última utilizada como um descritor livre, sem a utilização de aspas. Todas as palavraschave fazem parte dos Descritores em Ciências da Saúde (DeCS). 
Utilizaram-se os seguintes critérios de inclusão: artigos publicados em espanhol, inglês e português, no período de 2013 a 2017. Foram excluídos da pesquisa os artigos em forma de apostilas, pois não atendiam os critérios necessários, reforçando a importância da busca de evidências científicas sobre a temática.

Para coleta de dados foi elaborado um formulário, pelo próprio autor, contendo o título dos artigos, os nomes dos pesquisadores, informações sobre os periódicos (volume, número, páginas, ano e revista publicada) e os principais destaques. Posteriormente, os dados foram transmitidos para um quadro sinóptico com as mesmas informações para realização da síntese dos estudos.

Após leitura dos artigos científicos incluídos na revisão integrativa, ocorreu análise descritiva, por meio de cálculo percentual e apresentações de valores absolutos em tabelas. No momento posterior, os dados foram analisados por meio da síntese dos diálogos dos autores e discutidos com base na literatura pertinente. Os estudos foram agrupados por similaridade de conteúdo, evidenciando uma única categoria: $O$ enfermeiro no programa de gerenciamento do uso de antimicrobianos.

A Figura 1 demonstra os passos percorridos para seleção dos artigos que fizeram parte da amostra do estudo.

\section{RESULTADOS}

Neste estudo, foram incluídos seis artigos científicos, que atenderam os critérios de inclusão previamente estabelecidos. Todos foram publicados na língua inglesa e divulgados na base de dados MEDLINE. O Quadro 1 apresenta a síntese dos seis artigos que compuseram a amostra.

Do total de artigos sobre o assunto em questão, verificouse que a metade $(50,0 \%)$ foi publicada em revistas científicas que tiveram fator de impacto avaliado acima de 2,0, destacando os periódicos: Clinical Infectious Diseases (8,216), BMC

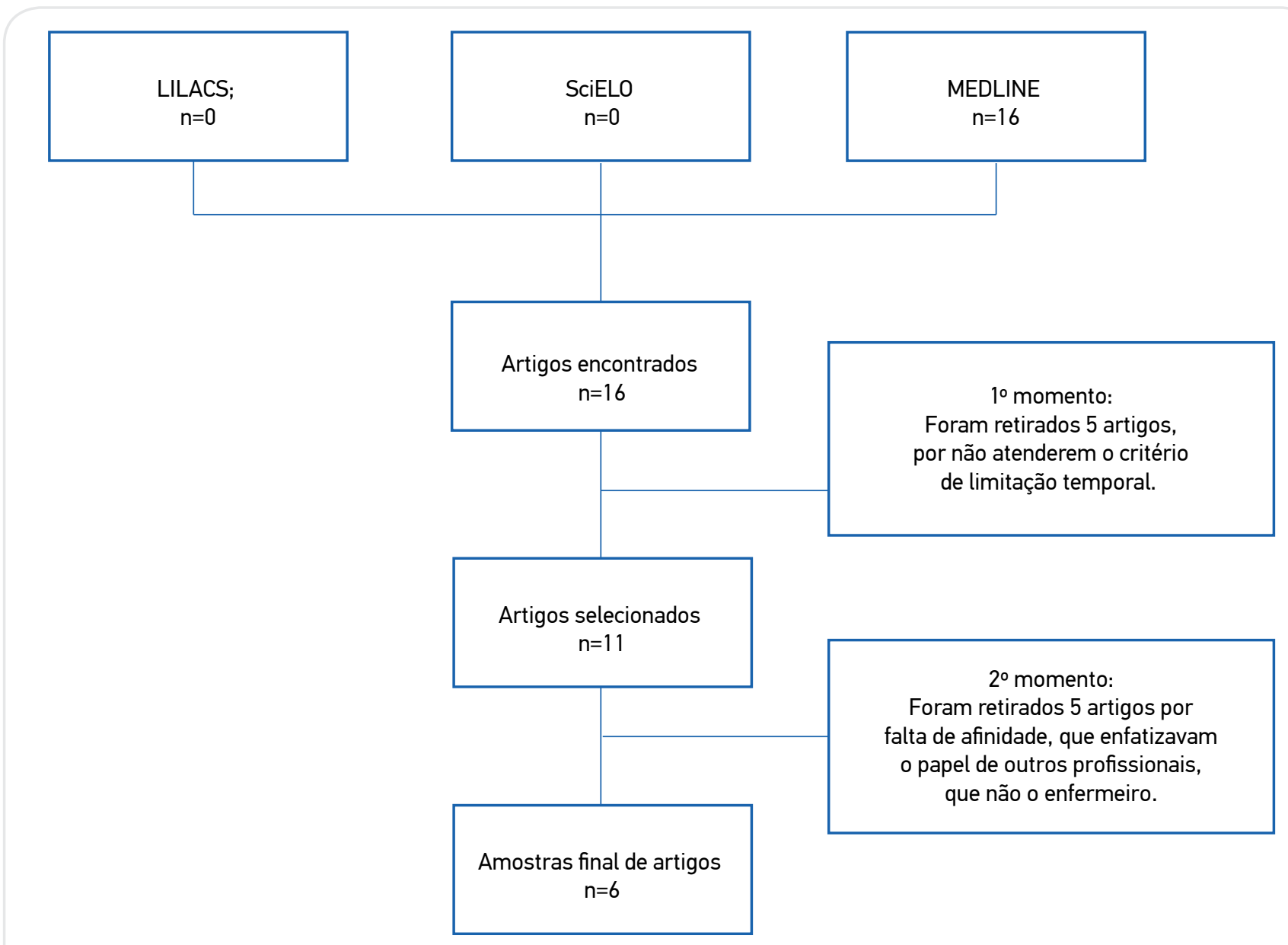

LILACS: Literatura Latino-americana e do Caribe em Ciências da Saúde; SciElo: Scientific Electronic Library Online; MEDLINE: Medical Literature Analysis and Retrieval System Online.

Figura 1. Busca literária de artigos científicos. 
Medical Informatics and Decision Making $(2,288)$ e American Journal of Infection Control $(2,209)$. (Quadro 2)

Em relação à delimitação temporal, observa-se nesta revisão integrativa que dois $(33,3 \%)$ dos artigos selecionados são do ano de 2013; dois (33,3\%) de 2014; um (16,67\%) de 2015 e um (16,67\%) de 2016 (Tabela 1). Ainda no que tange ao recorte temporal, foi notória a ausência de estudos brasileiros publicados nas bases de dados selecionadas, predominando estudos internacionais.

Quanto aos temas abordados, todos os artigos reconheceram o enfermeiro como peça fundamental para o PGUA. Os estudos destacaram as atividades executadas por esse profissional como essenciais para redução da resistência antimicrobiana, bem como para otimização terapêutica dos tratamentos infecciosos. Destacaram-se atividades fundamentais exercidas por enfermeiros, tais como a revisão diária das prescrições médicas e a administração dos antimicrobianos com indicação, dose e duração adequadas.
Quadro 2. Fator de impacto das revistas científicas avaliadas para o estudo.

\begin{tabular}{|l|c|}
\hline Periódico & Fator de impacto \\
\hline Clinical Infectious Diseases & 8,216 \\
\hline $\begin{array}{l}\text { BMC Medical Informatics } \\
\text { and Decision Making }\end{array}$ & 2,288 \\
\hline American Journal of Infection Control & 2,209 \\
\hline $\begin{array}{l}\text { Journal of the American } \\
\text { Association of Nurse Practitioners }\end{array}$ & 0,931 \\
\hline Journal of Continuing Education in Nursing & 0,880 \\
\hline Nursing Standard & 0,080 \\
\hline
\end{tabular}

Tabela 1. Distribuição dos estudos, segundo o ano de publicação.

\begin{tabular}{|l|c|c|}
\hline Ano de publicação & Número & Percentagem \\
\hline 2013 & 2 & 33,3 \\
\hline 2014 & 2 & 33,3 \\
\hline 2015 & 1 & 16,7 \\
\hline 2016 & 1 & 16,7 \\
\hline 2017 & - & - \\
\hline
\end{tabular}

Quadro 1. Quadro sinóptico com a síntese dos artigos selecionados para o estudo.

\begin{tabular}{|c|c|c|c|}
\hline Nome do artigo & Autores & $\begin{array}{l}\text { Revista, ano, } \\
\text { volume, número e } \\
\text { páginas }\end{array}$ & Principais destaques \\
\hline $\begin{array}{l}\text { The critical role of the staff nurse } \\
\text { in antimicrobial stewardship: } \\
\text { unrecognized, } \\
\text { but already there }^{10}\end{array}$ & $\begin{array}{l}\text { Olans RN, Olans RD, } \\
\text { DeMaria A Jr. }\end{array}$ & $\begin{array}{l}\text { Clin Infect Dis. } \\
\text { 2016;62(1):84-9 }\end{array}$ & $\begin{array}{l}\text { O enfermeiro é um profissional que sempre } \\
\text { desempenhou inúmeras funções essenciais para } \\
\text { o sucesso da gestão antimicrobiana, tais como } \\
\text { monitorização contínua do status do paciente e dos } \\
\text { avaliadores da resposta terapêutica antimicrobiana. }\end{array}$ \\
\hline $\begin{array}{l}\text { Defining a role for nursing } \\
\text { education in staff nurse } \\
\text { participation in antimicrobial } \\
\text { stewardship } 11\end{array}$ & $\begin{array}{l}\text { Olans RD, Nicholas } \\
\text { PK, Hanley D, } \\
\text { DeMaria A Jr. }\end{array}$ & $\begin{array}{l}\text { J Contin Educ Nurs. } \\
2015 ; 46(7): 318-21\end{array}$ & $\begin{array}{c}\text { O papel do enfermeiro no PGUA não está claramente } \\
\text { definido, necessitando de intervenções educacionais } \\
\text { para melhorar a conscientização acerca dos } \\
\text { componentes do programa. }\end{array}$ \\
\hline $\begin{array}{l}\text { The urgent need for nurse } \\
\text { practitioners to lead } \\
\text { antimicrobial stewardship in } \\
\text { ambulatory health care }{ }^{12}\end{array}$ & Manning ML & $\begin{array}{l}\text { J Am Assoc } \\
\text { Nurse Pract. } \\
2014 ; 26(8): 411-3\end{array}$ & $\begin{array}{c}\text { No PGUA, é essencial que os profissionais de } \\
\text { enfermagem assumam a liderança ao abordar as } \\
\text { implicações terapêuticas para os pacientes e para } \\
\text { sua prática assistencial. }\end{array}$ \\
\hline $\begin{array}{l}\text { Participatory eHealth } \\
\text { development to support nurses } \\
\text { in antimicrobial stewardship }{ }^{13}\end{array}$ & $\begin{array}{l}\text { Wentzel J, Van Velsen } \\
\text { L, Van Limburg M, de } \\
\text { Jong N, Karreman J, } \\
\text { Hendrix R, et al. }\end{array}$ & $\begin{array}{l}\text { BMC Med Inform } \\
\text { Decis Mak. } \\
\text { 2014;14:45. }\end{array}$ & $\begin{array}{c}\text { Para realizar atividades complexas relacionadas aos } \\
\text { antimicrobianos, os enfermeiros precisam consultar } \\
\text { várias fontes de informação em uma infinidade de } \\
\text { ocasiões, principalmente porque as informações } \\
\text { não estão estruturadas para corresponder às tarefas } \\
\text { desse profissional. }\end{array}$ \\
\hline $\begin{array}{l}\text { Antimicrobial stewardship: } \\
\text { the role of the nurse }{ }^{14}\end{array}$ & $\begin{array}{l}\text { Ladenheim D, } \\
\text { Rosembert D, Hallam } \\
\text { C, Micallef C }\end{array}$ & $\begin{array}{c}\text { Nurs Stand. 2013; } \\
\text { 28(6):46-9. }\end{array}$ & $\begin{array}{l}\text { O papel do enfermeiro é fundamental para garantir } \\
\text { o uso correto dos antimicrobianos por meio da } \\
\text { administração medicamentosa correta, contribuindo } \\
\text { para redução da resistência antimicrobiana. }\end{array}$ \\
\hline $\begin{array}{l}\text { Improving antibiotic stewardship } \\
\text { by involving nurses }{ }^{15}\end{array}$ & $\begin{array}{c}\text { Gillespie E, Rodrigues } \\
\text { A, Wright L, Williams } \\
\text { N, Stuart RL }\end{array}$ & $\begin{array}{l}\text { Am J Infect Control. } \\
\text { 2013;41(4):365-7. }\end{array}$ & $\begin{array}{l}\text { Os enfermeiros são fundamentais para administração } \\
\text { de medicamentos prescritos pela equipe médica. } \\
\text { O PGUA deve envolver esses profissionais com o } \\
\text { intuito de melhorar a administração antimicrobiana no } \\
\text { ambiente hospitalar. }\end{array}$ \\
\hline
\end{tabular}

PGUA: Programa de Gerenciamento do Uso de Antimicrobianos. 


\section{DISCUSSÃO}

\section{0 enfermeiro no programa de gerenciamento do uso de antimicrobianos}

As prescrições médicas classificadas como inadequadas ou desnecessárias podem variar entre 20 e 50\%, justificando a adoção do PGUA como estratégia para garantia do efeito fármaco-terapêutico eficaz e para o controle do consumo de antibióticos nos serviços de saúde ${ }^{16}$. Com isso, o uso indiscriminado dessas substâncias poderá ser reduzido ao máximo, impactando no declínio da resistência antimicrobiana que ameaça a saúde pública mundial.

O PGUA envolve uma abordagem multifacetada de políticas, diretrizes, estratégias educacionais e outras abordagens que asseguram os melhores desfechos clínicos. O controle e a vigilância rigorosa dos antimicrobianos visam reduzir ou eliminar riscos potenciais, que impactam no tratamentos anti-infeccioso dos pacientes ${ }^{3}$.

O manejo adequado dos antimicrobianos nos serviços de saúde pode reduzir, consideravelmente, as IRAS causadas por microrganismos multirresistentes ${ }^{16}$. É imprescindível a formação de um time interdisciplinar que seja responsável pela execução das ações de melhoria relacionadas ao PGUA, capacitando os gestores em saúde e divulgando feedbacks aos envolvidos ${ }^{3,5-8}$.

A diretriz nacional para elaboração do Programa de Gerenciamento do Uso de Antimicrobianos em Serviços de Saúde, lançado pela ANVISA em 2017, sugere a equipe de enfermagem como um dos representantes para formação de um time gestor. Esse time deve coordenar ações administrativas e gerais, estabelecendo avaliações e controle sistemáticos para o cumprimento do plano nas diferentes unidades de um hospital. Esse documento cita, também, o time operacional, que é responsável pela elaboração, execução e monitoramento das ações do Programa de Gerenciamento, devendo ser formado, no mínimo, pelos seguintes componentes do time gestor: infectologista ou médico com expertise em doenças infecciosas; farmacêutico clínico, preferencialmente com expertise em doenças infecciosas e uso de antimicrobianos; enfermeiro da Comissão de Controle de Infecção Hospitalar (CCIH) e microbiologista clínico ${ }^{3}$.

O enfermeiro possui papel peculiar no cuidado direto ao paciente, desenvolvendo diversas atividades assistenciais complexas, entre elas a administração de medicamentos. Um estudo mostrou a importância desse profissional como uma das lideranças frente ao PGUA, abordando as várias implicações relacionadas à administração dos antimicrobianos para a prática assistencial do enfermeiro ${ }^{12}$.
Nesse sentido, a importância da enfermagem na racionalização da antibioticoterapia, por meio da administração medicamentosa, deve ser mais explorada nos serviços de saúde $^{10,14,15}$. É importante ressaltar que o próprio enfermeiro precisa se enxergar dentro do PGUA, valorizando seus conhecimentos como educador e seu suporte informante das práticas associadas à gestão antimicrobiana.

Embora o papel do enfermeiro não tenha sido formalmente reconhecido frente ao PGUA ou definido na literatura médica, é importante elucidar que o profissional sempre desempenhou inúmeras funções essenciais para o sucesso do programa. São considerados comunicadores centrais do status do paciente, avaliando durante 24 horas os efeitos e/ ou os possíveis eventos adversos relacionados à antibioticoterapia ${ }^{10}$.

Um estudo avaliou 900 artigos científicos sobre gestão antimicrobiana hospitalar e os autores identificaram que somente 11 haviam sido publicados em revistas de enfermagem $^{11}$. Nesse caso, a maioria encontrava-se disponível em periódicos médicos, de farmácia, microbiologia e outros, enfatizando a falta de estudos elaborados por enfermeiros sobre a temática.

Pesquisa realizada com 180 enfermeiros mostrou que os profissionais possuem grande destaque, principalmente na identificação das reações adversas e obtenção das culturas bacteriológicas antes do início dos antimicrobianos, e participação ativa na educação em saúde relacionada à temática. No entanto, os autores desse estudo salientaram oportunidades de melhoria em alguns aspectos, como, por exemplo, na revisão dos resultados de microbiologia para determinar a adequação do antibiótico e falhas na inserção do enfermeiro em rodadas de discussão da equipe multidisciplinar ${ }^{17}$.

Outro aspecto relevante é a educação dos profissionais de saúde, dos pacientes, dos acompanhantes e dos cuidadores para o sucesso do PGUA, sendo um fator essencial que favorece a conscientização e estimula positivamente o time operacional. O processo educacional para o envolvimento de todos promove o uso correto dos antimicrobianos e favorece a gestão terapêutica eficaz de pacientes com infecção, potencializando os desfechos favoráveis com menores eventos adversos ${ }^{3}$.

No entanto, os enfermeiros ainda precisam aprimorar seus conhecimentos acerca dos componentes do PGUA ${ }^{11}$. Estudo mostrou que $98 \%$ no total de 210 enfermeiros ainda relatam carência de formação específica relacionada aos antimicrobianos. Infere-se que, a educação sobre a temática não deve se limitar à graduação, necessitando ser expandida para os locais que envolvem a prática assistencial ao paciente ${ }^{18}$. 
Além da necessidade de aprimorar o conhecimento dos enfermeiros em relação o PGUA, autores ${ }^{13}$ consideram que os profissionais ainda precisam consultar várias fontes de informação em uma infinidade de ocasiões, principalmente porque os dados específicos do programa não estão estruturados para corresponder às tarefas do profissional. Assim, percebeu-se a necessidade de criar tecnologias que favoreçam a incorporação dos protocolos médicos às rotinas de trabalho do enfermeiro, reduzindo retrabalho $\mathrm{e}$ otimizando as boas práticas do uso de antimicrobianos.

Com base na leitura minuciosa dos artigos selecionados, foi notória a importância da participação do enfermeiro na racionalização do uso dos antimicrobianos. Destaca-se que suas atribuições específicas contribuem para a efetividade das ações do PGUA, em que suas preocupações quanto à seleção do antibiótico, vias de administração, dosagem e duração dos tratamentos favorecem os melhores desfechos clínicos, com mínimo risco potencial.

\section{CONSIDERAÇÕES FINAIS}

O Programa de Gerenciamento do Uso de Antimicrobianos (PGUA) otimiza a terapia medicamentosa e diminui a resistência dos microrganismos aos antibióticos. Para que o programa seja executado com êxito, as diretrizes de prevenção e controle de infecções devem ser adotadas em conjunto, de modo a reduzir a transmissão dos patógenos aos pacientes em atendimento em uma unidade assistencial.

Para execução das políticas e normas relacionadas ao PGUA, é essencial que haja formação de um time interdisciplinar, composto por diversos profissionais. Embora o enfermeiro não tenha sido reconhecido por alguns estudos como membro da gestão antimicrobiana, a sua participação promove otimização terapêutica, bem como uso racional dos antimicrobianos.

O objetivo principal do estudo foi alcançado, e por meio dessa revisão integrativa, foi possível conhecer o papel do enfermeiro e sua importância frente ao PGUA. Vale a pena destacar os demais profissionais que compõem o time gestor, tais como infectologista, farmacêutico, enfermeiro da CCIH e microbiologista clínico, contribuindo, de forma mútua, para a gestão terapêutica, com desfechos favoráveis e menores eventos adversos ao paciente. No entanto, mais pesquisas com outras metodologias e novos enfoques são fundamentais para melhor compreensão da temática estudada.

\section{REFERÊNCIAS}

1. Centers for Disease Control and Prevention (CDC). Antibiotic resistance threats in the United States, 2013 [Internet]. Atlanta: Centers for Disease Control and Prevention; 2013 [acessado em 8 jun. 2018]. Disponivel em: https://www.cdc.gov/drugresistance/threat-report-2013/pdf/ ar-threats-2013-508.pdf

2. European Centre for Disease Prevention and Control. Factsheet for experts: antimicrobial resistance [Internet]. European Centre for Disease Prevention and Control; 2008 [acessado em 8 jun. 2018]. Disponível em: https://ecdc.europa.eu/en/antimicrobial-resistance/ facts/factsheets/experts

3. Brasil. Agência Nacional de Vigilância Sanitária (ANVISA). Diretriz Nacional para Elaboração de Programa de Gerenciamento do Uso de Antimicrobianos em Serviços de Saúde. Brasília [Internet]. Brasil: Agência Nacional de Vigilância Sanitária; 2017 [acessado em 9 jun. 2018]. Disponível em: https://www20.anvisa.gov.br/ segurancadopaciente/index.php/publicacoes/item/diretriz-nacionalpara-elaboracao-de-programa-de-gerenciamento-do-uso-deantimicrobianos-em-servicos-de-saude

4. Brasil. Ministério da Saúde (MS). Portaria n².616, de 12 de maio de 1998. Dispõe sobre diretrizes e normas para prevenção e o controle das infecções hospitalares [Internet]. Brasília: Ministério da Saúde; 1998 [acessado em 9 jun. 2018]. Disponível em: http://bvsms.saude. gov.br/bvs/saudelegis/gm/1998/prt2616_12_05_1998.html
5. World Health Organization. Global strategy for containment of antimicrobial resistance [Internet]. Genebra: World Health Organization; 2001 [acessado em 10 jun. 2018]. Disponivel em: http://www.who. int/drugresistance/WHO_Global_Strategy_English.pdf

6. Pollack LA, Srinivasan A. Core elements of hospital antibiotic stewardship programs from the centers for disease control and prevention. Clin Infec Dis [Internet]. 2014 [acessado em 10 jun. 2018];59(Supl. 3):s97s100. Disponível em: http://dx.doi.org/10.1093/cid/ciu542

7. Perez KK, Olsen RJ, Musick WL, Cernoch PL, Davis JR, Peterson LE, et al. Integrating rapid diagnostics and antimicrobial stewardship improves outcomes in patients with antibiotic-resistant $\mathrm{Gram}$-negative bacteremia. J Infect [Internet]. 2014 [acessado em 10 jun. 2018];69(3):216-25. Disponivel em: http://dx.doi.org/10.1016/j.jinf.2014.05.005

8. Centers for Disease Control and Prevention (CDC). Core Elements of Hospital Antibiotic Stewardship Programs [Internet]. Atlanta: US Department of Health and Human Services, CDC; 2014 [acessado em 10 jun. 2018]. Disponível em: http://www.cdc.gov/getsmart/ healthcare/implementation/core-elements.html

9. Mendes KS, Silveira RCCP, Galvão CM. Revisão integrativa: método de pesquisa para a incorporação de evidências na saúde e na enfermagem. Texto Context Enferm [Internet]. 2008 [acessado em 10 jun. 2018];17(4):75864. Disponivel em: http://dx.doi.org/10.1590/S0104-07072008000400018 
10. Olans RN, Olans RD, de Maria Jr. A. The critical role of the staff nurse in antimicrobial stewardship: unrecognized, but already there. Clin Infect Dis [Internet]. 2016 [acessado em 10 jun. 2018];62(1):84-9. Disponível em: http://dx.doi.org/10.1093/ cid/civ697

11. Olans RD, Nicholas PK, Hanley D, DeMaria A Jr. Defining a role for nursing education in staff nurse participation in antimicrobial stewardship. J Contin Educ Nurs [Internet]. 2015 [acessado em 9 jun. 2018];46(7):318-21. Disponível em: http://dx.doi. org/10.3928/00220124-20150619-03

12. Manning ML. The urgent need for nurse practitioners to lead antimicrobial stewardship in ambulatory health care. J Am Assoc Nurse Pract [Internet]. 2014 [acessado em 10 jun. 2018];26(8):411-3. Disponível em: http://dx.doi. org/10.1002/2327-6924.12150

13. Wentzel J, Van Velsen L, Van Limburg M, de Jong N, Karreman J, Hendrix R, et al. Participatory eHealth development to support nurses in antimicrobial stewardship. BMC Med Inform Decis Mak [Internet]. 2014 [acessado em 9 jun. 2018];14(45). Disponível em: https://doi. org/10.1186/1472-6947-14-45
14. Ladenheim D, Rosembert D, Hallam C, Micallef C. Antimicrobial stewardship: the role of the nurse. Nurs Stand. 2013;28(6):46-9. http://dx.doi.org/10.7748/ns2013.10.28.6.46.e7802

15. Gillespie E, Rodrigues A, Wright L, Williams N, Stuart RL. Improving antibiotic stewardship by involving nurses. Am J Infect Control. 2013;41(4):365-7. https://doi.org/10.1016/j.ajic.2012.04.336

16. Fridkin S, Baggs J, Fagan R, Magill S, Pollack LA, Malpiedi P, et al. Vital signs: improving antibiotic use among hospitalizated patients. MMWR Morb Mortal Wkly Rep [Internet]. 2014 [acessado em 12 jun. 2018];63(9):194-200. Disponível em: https://www.cdc.gov/mmwr/ preview/mmwrhtml/mm6309a4.htm

17. Monsees E, Popejoy L, Jackson MA, Lee B, Goldman J. Integrating staff nurses in antibiotic stewardship: Opportunities and barriers. Am J Infect Control [Internet]. 2018 [acessado em 9 jun. 2018];46(7):73742. Disponivel em: https://doi.org/10.1016/j.ajic.2018.03.028

18. AberaB, Kibret M, Wondemagegn M. Knowledge and beliefs on antimicrobial resistance among physicians and nurses in hospitals in Amhara Region, Ethiopia. BMC Pharmacol Toxicol [Internet]. 2014 [acessado em 11 jun. 2018];15:26. Disponivel em: http://dx.doi.org/10.1186/2050-6511-15-26 